\title{
CHEST What's hot that the other's CLINIC got
}

\author{
Janice Higginson
}

\section{UPDATING THE DEFINITION OF BRONCHOPULMONARY DYSPLASIA}

This retrospective cohort study (JAMA Paeditr. Published Online First: 23 Jan 2017. http://dx.doi.org/10.1001/jamapediatrics. 2016.4141) included $80 \%$ of neonates born in Canada at less than 29 weeks gestation from January 2010 to September 2011. The aim was find out which of six definitions of bronchopulmonary dysplasia are most predictive of respiratory morbidity or neurosensory deficit, as measured at 18-21 months corrected age. The group then analysed at what gestational age application of the definition would be most accurate. They showed that the existing definition giving the best predictive value was the use of oxygen or respiratory support at 36 weeks gestational age, but that accuracy could be improved by applying this definition at 40 weeks. The application of a single definition that is appropriate to contemporary neonatal medicine will surely be beneficial to the specialty leading to improvements in patient care.

\section{DISTRESS CAUSES CANCER?}

Batty et al (BMJ 2017;356:j108) used raw data from 16 studies contributing to Health Survey for England and Scottish Health Service from 1994 to 2008 to produce a huge prospective cohort study. Baseline psychological distress was quantified using GHQ-12 and the cohorts observed for cancer incidence. When the highest level of distress was compared with the lowest there was a statistically significant relationship with high distress and being female, obese, smoking, leaving school earlier and, surprisingly, drinking alcohol, less. When the data sets for all 16 studies were examined the HRs for different cancer sites showed a more convincing association between high distress and cancer mortality, particularly for colorectal, prostate, oesophageal and pancreas cancers, and leukaemia. This is a controversial area and one in which the causative and associative factors are difficult to

Correspondence to Dr Janice Higginson, ST5 Freeman Hospital, Newcastle-Upon-Tyne, Northumberland NE7 7DN, UK; janice.higginson@nhs.net distinguish, even in a large population study such as this. Further work is needed if the causative links between psychological distress, inflammation and cancer are to be made.

\section{INTRAVENOUS ZANAMAVIR IN SEVERE INFLUENZA}

Influenza has already caused a high level of clinical burden in hospitals this winter. In this multinational, multicentre, randomised, double-blind, double-dummy study (Lancet Resp Med 2017;5:135-46) intravenous zanamivir $600 \mathrm{mg}$ was compared with intravenous zanamivir $300 \mathrm{mg}$ or oral/NG oseltamivir $75 \mathrm{mg}$ twice daily. Six hundred and fifteen patients were recruited of whom 488 were influenzapositive and $40 \%$ were in an intensive care unit. Results for the influenzapositive group showed no significant difference in time to clinical response in any of the treatment arms, and this remained true when looking at patients with symptom onset $\leq 4$ days, or mechanical ventilation or intensive care at baseline. Median time to no detectable RNA was 4 days in all treatment arms. Some treatmentemergent mutations were detected, most notably four patients in the oseltamivir group who had influenza A H1N1pdm09. The authors concluded than zanamivir at either dose is not inferior to oseltamivir and represents a treatment option for patients requiring intravenous therapy.

\section{EGFR DETECTION THROUGH BLOOD SAMPLING}

Yi-Long Wu et al (Br J Canner 2017; 116:175-85.) publish the results from LL3 and LL6 phase 3 trials. Both trials took patients with performance status $0-1$ and stage 111B/IV non-small cell lung cancer with tissue positive for any EGFR mutation. LL3 used afatinib versus cisplatin and pemetrexed and detected EGFR circulating free DNA (cf-DNA) in serum samples; LL6 was afatinib versus cisplatin and gemcitabine and detected EGFR using plasma samples. Concordance between tissue and serum samples in LL3 and LL6 was $28.6 \%$ and $60.5 \%$, respectively. cf-DNA-positive patients more frequently had a performance status of 1 rather than 0 , greater tumour burden and statistically significantly more metastatic sites-metastases to the bone and liver. This suggests more aggressive and advanced tumours leak EGFR into blood at higher levels; this theory is reflected in the results showing a generally shorter progression-free survival and overall survival for those who were cf-DNA-positive. As shown in previous trials, afatinib significantly improved outcomes over platinum-based chemotherapy for all EGFR-positive patients. Further work is needed to make the cf-DNA assays an effective replacement for tissue biopsy, but if successful, could potentially reduce the number and size of biopsies required, shortening the patient pathway.

\section{ROLE OF GRAFT DONOR-SPECIFIC ANTIGEN MEASUREMENT POST LUNG TRANSPLANT}

Antibody mediation rejection (AMR) is a significant risk to graft survival in lung transplant recipients and is associated with development of bronchiolitis obliterans. Donor-specific antibodies (DSAs) from serum (sDSA) are routinely measured post transplant to detect AMR and transbronchial biopsies are routinely performed to screen for histological signs of rejection. Visenting et al (J Heart Lung Transplant 2016;35:1418-26) aimed to increase the clinical use of these monitoring procedures. They retrospectively examined biopsy samples for DSAs found within the grafted lung (gDSA). Results showed that graft survival was significantly reduced at 1 year in gDSA-positive than gDSA-negative samples; there was no statistically significant difference in graft survival between sDSA-positive and sDSAnegative samples. The authors recognise that this was a single-centre retrospective study and that further work needs to be done, but this is an interesting area of development and the authors acknowledge that there are areas of posttransplant immunology that are not fully understood.

\section{Competing interests None declared.}

Provenance and peer review Commissioned; internally peer reviewed.

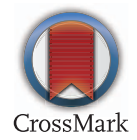

To cite Higginson J. Thorax 2017;72:388.

Published Online First 3 March 2017

Thorax 2017;72:388

doi:10.1136/thoraxjnl-2017-210111 